\title{
An experimental investigation of the influence of intermittent and continuous operating modes on the thermal behaviour of a full scale geothermal energy pile
}

\author{
Mohammed Faizal $^{a}$, Abdelmalek Bouazza $^{a}$, Rao M. Singh ${ }^{\mathrm{b}}$ \\ ${ }^{a}$ Department of Civil Engineering, 23 College Walk, Monash University, Melbourne, Vic. 3800, Australia \\ ${ }^{\mathrm{b}}$ Department of Civil and Environmental Engineering, University of Surrey, Surrey, GU2 7XH, United Kingdom
}

\begin{abstract}
Heat pumps connected to geothermal energy piles have variable operating hours. These variable hours can affect the thermal behaviour of these piles. Some key findings are presented for intermittent (16 hour) and continuous (24 hour) cooling modes in this abstract. It is found that the 16 hour cooling mode is more beneficial in terms of energy extraction, ground recovery, pile temperature and strain recovery.
\end{abstract}

\section{Introduction and background}

Many studies conducted on variable operating hours of heat pumps have mostly focused on the variation of heat exchange and its effect on ground temperatures. Li et al. [1] simulated the effect of intermittent and continuous operation on the underground temperature fields of boreholes and piles. The continuous mode always had higher impact on the ground temperature compared to intermittent mode. You et al. [2] conducted experimental studies on piles for continuous and 24 hour intermittent modes and found that heat transfer was higher in intermittent mode compared to continuous mode. In another study, Park et al. [3] had concluded that intermittent operation is good for ground recovery as well as for better heat exchange. Jalaluddin et al. [4] experimentally studied the heat exchange in a steel pile, for continuous (24 hour) and discontinuous (2 hour) modes. They found that heat exchange rate for 2 hour operation was always higher.

While it is clear from previous studies that intermittent modes improve heat exchange due, studies on the reaction of internal pile temperatures are limited. Also, the response of thermal strains has not been studied. The studies of thermal strains will show how elastic the pile response is for stop-run operations compared to continuous operations. Also, continuous operation may have continuous thermal stresses at the concrete/soil interface due to induced thermal strains in the pile. The main objective is thus to investigate the thermal behaviour of a full scale geothermal energy pile under intermittent (16 hours per day) and continuous (24 hours per day) modes of operations. Some key findings are presented in this abstract.

\section{Experimental setup and procedure}

The experiments were carried out on an instrumented, full-scale geothermal energy pile at Monash University, Melbourne, Australia, for 480 hours (20 days). A detailed description of the installation is given by Wang et al [5]. A chiller was used to circulate water at $5{ }^{\circ} \mathrm{C}$ and $15 \mathrm{~L} / \mathrm{min}$ in the system.

\section{Results and discussions}

It is found that more energy is extracted in 16 hour (8 hour recovery) mode due to ground temperature recovery (Fig.1). The 24 hour mode rapidly reduces the temperature gradient between the inlet fluid and ground temperature, and thus, energy extraction reduces with time. Therefore, the intermittent

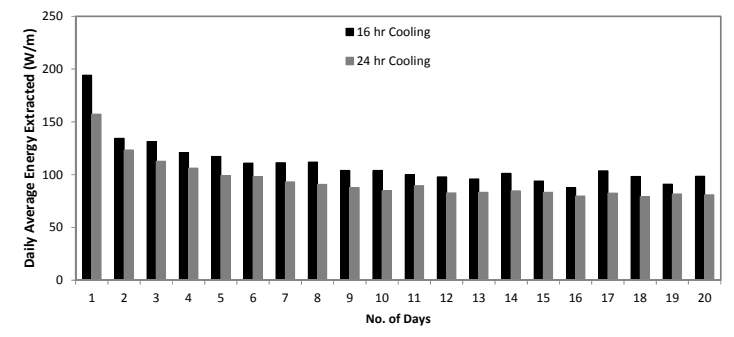

Fig.1. Average daily energy extracted for both cooling modes. 
mode is beneficial for energy extraction compared to continuous mode. The 8 hours ground recovery time in 16 hour mode delays ground recovery (Fig.2). Thus, the intermittent mode of operation is beneficial for long term ground recovery as well.
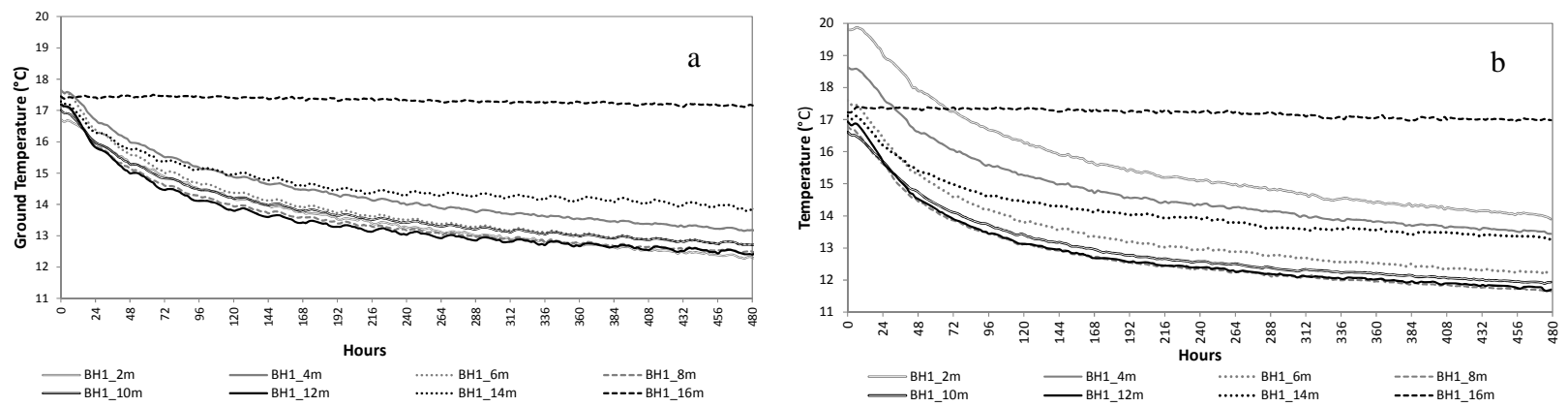

Fig.2. Ground temperatures a) 16 hour mode b) 24 hour mode.

Pile temperature drop is initially higher for both modes due to high thermal gradient between inlet fluid and soil (Fig. 3). An immediate temperature change is observed when the chiller is switched off/on for 16 hour mode. After reaching equilibrium, peak temperatures at end of cooling/recovery for 16 hour mode is similar for all cycles, showing elastic response of the pile during operation. While there is a continuous pile temperature of approximately $6.9^{\circ} \mathrm{C}$ in the 24 hour mode for any given day after thermal equilibrium, there is a transient temperature change from approximately $10^{\circ} \mathrm{C}$ to $6.9^{\circ} \mathrm{C}$ in the 16 hour mode everday. Thus, 16 hour mode gives lower overall thermal loading compared to 24 hour mode.
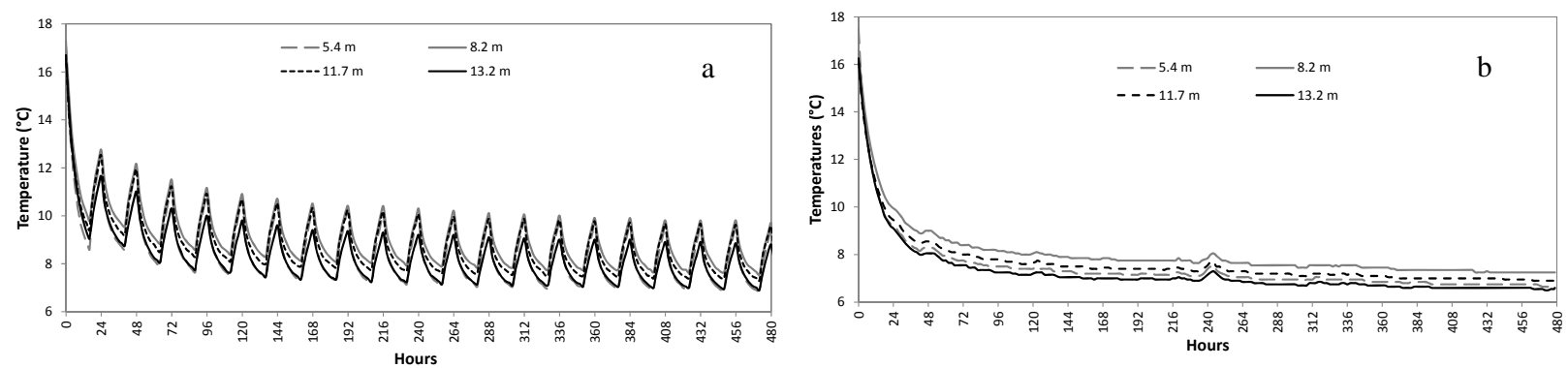

Fig.3. Pile transient temepratures a) 16 hour cooling mode b) 24 hour cooling mode.

Temperatures at end of cooling and end of recovery for 16 hour mode, and at every 24 hours for continuous cooling mode are shown with depth (Fig.4). Pile temperatures reduce and approach steady state gradually. After reaching thermal equilibrium, the pile temperatures return to stable peak temperatures at end of cooling and recovery cycles for 16 hour mode. The 24 hour mode is under continuous thermal loads without any recovery time, thus the difference between temperatures for consecutive days is lower compared to 16 hour mode.

Thermal strains follow temperature trends (Fig.4). After reaching thermal equilibrium, an immediate response in thermal strains is observed when the chiller is switched off/on in 16 hour mode. The peak strains at end of cooling/recovery for 16 hour mode is same for all cycles, showing elastic response of the pile during operation. Radial strains were found to be higher compared to axial strains. While there is a continuous thermal strain in the 24 hour mode for any given day after thermal equilibrium (-116 microstrains for axial), there is a transient change of thermal strains from approximately - 80 to - 116 microstrains (for axial) in the 16 hour mode for the same day. Thus, 16 hour will give lower overall thermal stresses in the pile compared to 24 hour mode.

Thermal strains at end of cooling and end of recovery for 16 hour mode, and at every 24 hours for 24 hour mode are shown against depth (Fig.5). Initial strain change is highest due to high temperature gradient between soil and inlet fluid. The thermal strains reduce and approach steady state gradually. After reaching equilibrium, thermal strains for 16 hour cooling return to stable peak values at end of cooling and recovery cycles. Since the 24 hour mode is under continuous thermal loads without any recovery time, the difference between thermal strains for consecutive days is lower compared to 16 hour mode. 

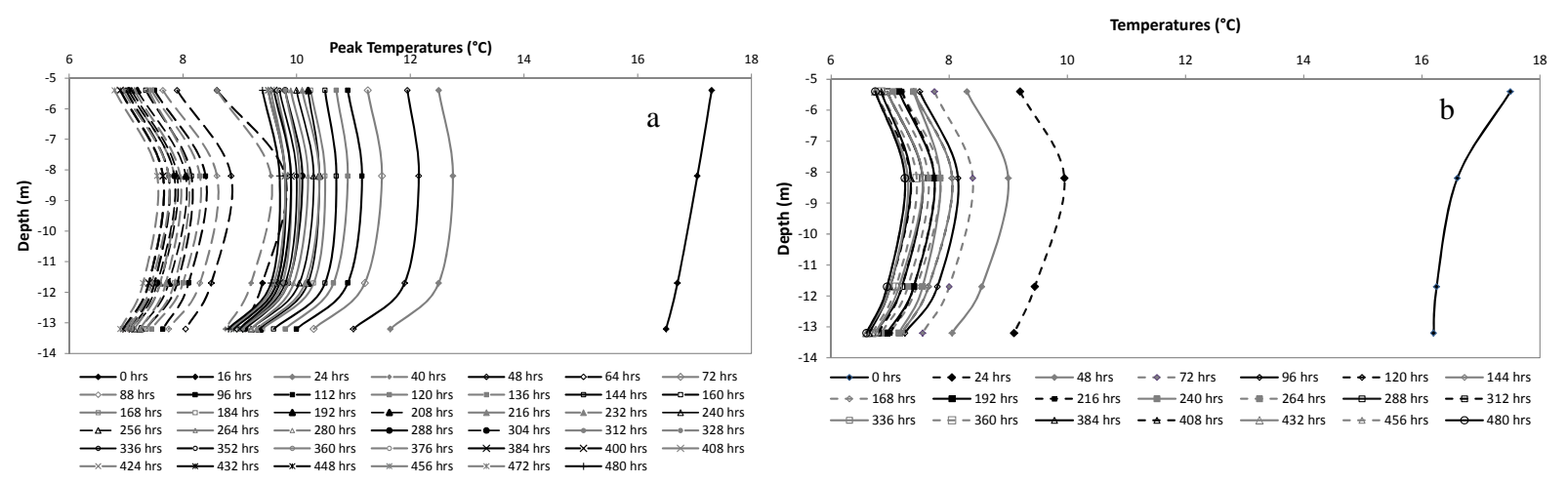

Fig.3. Pile temeprtaures with depth a) 16 hour cooling mode b) 24 hr cooling mode.
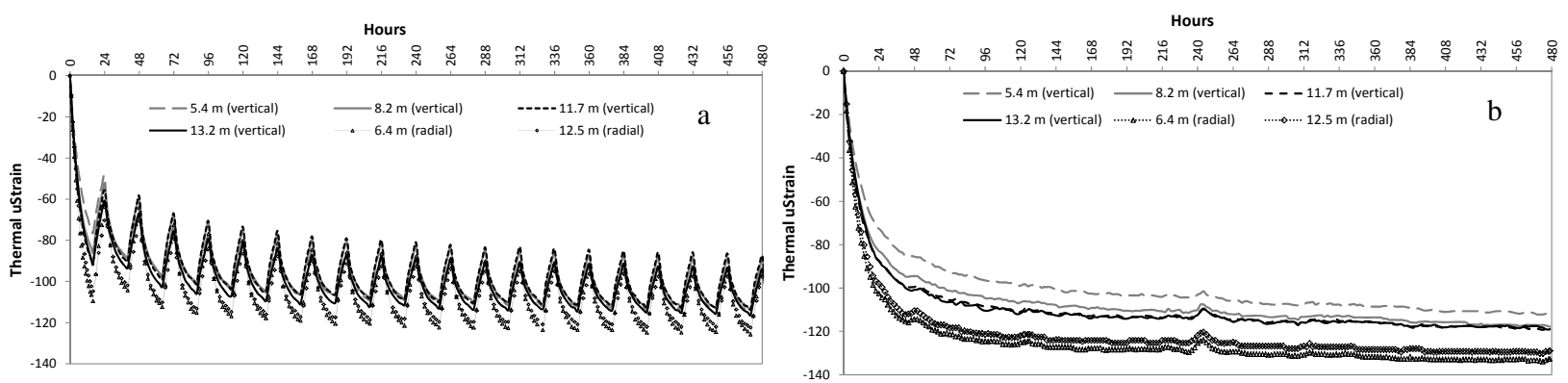

Fig.4. Transient thermal strains a) 16 hour cooling mode b) 24 hr cooling mode.
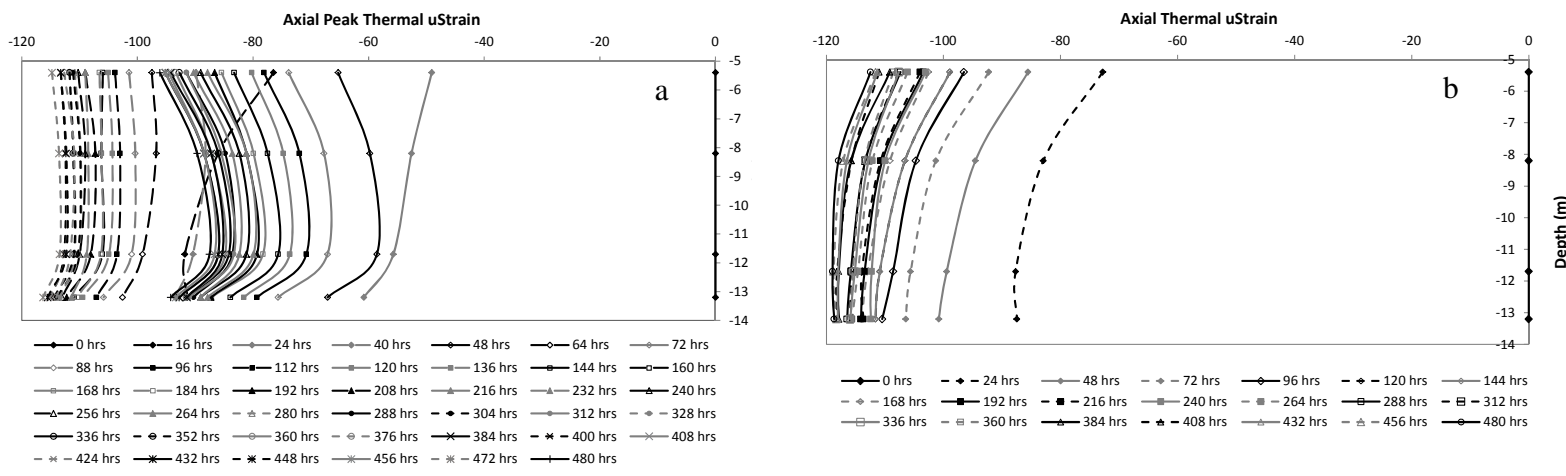

Fig.5. Axial thermal strains with depth a) 16 hour cooling mode b) 24 hr cooling mode.

\section{Conclusions}

A comparison of the energy extraction, pile and ground tempertaures and observed thermal strains has been done for a full scale geothermal energy pile, for intermittent and continuous (16 hour and 24 hour) cooling modes. It is found that the pile undergoes a thermoelastic behaviour during operation for a 16 hour cooling with 8 hour recovery. The maximum observed thermal strains for both cases are similar. Compared to 24 hour continuous mode, the 16 hour mode is concluded to be more beneficial in terms of energy extraction, ground temperature recovery, and maximum thermal strains.

\section{References}

[1] Li X, Chen Y, Chen Z, Zhao J. Thermal performances of different types of underground heat exchangers. Energ Buildings. 2006;38:543-7.

[2] You S, Cheng X, Guo H, Yao Z. In-situ experimental study of heat exchange capacity of CFG pile geothermal exchangers. Energ Buildings. 2014;79:23-31.

[3] Park H, Lee S-R, Yoon S, Choi J-C. Evaluation of thermal response and performance of PHC energy pile: Field experiments and numerical simulation. Applied Energy. 2013;103:12-24.

[4] Jalaluddin AM, Koutaro Tsubaki, Kentaro Yoshida. Thermal Performances Of Three Types Of Ground Heat Exchangers In Short-Time Period Of Operation. International Refrigeration and Air Conditioning Conference. Purdue2010.

[5] Wang B, Bouazza A, Singh R, Haberfield C, Barry-Macaulay D, Baycan S. Posttemperature Effects on Shaft Capacity of a Full-Scale Geothermal Energy Pile. J Geotech Geoenviron.2015, 141 (4):04014125. 